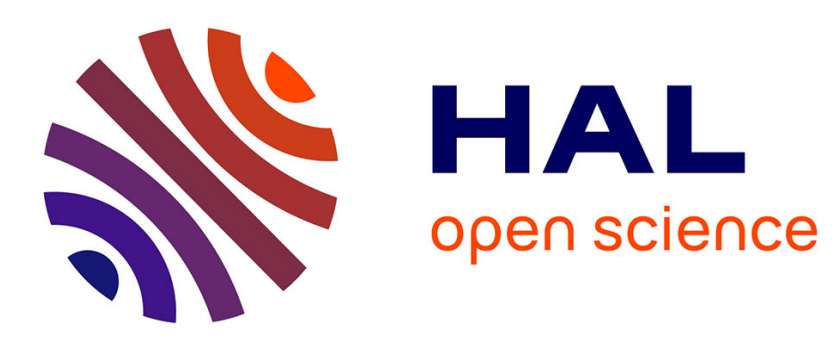

\title{
Simulation of the Dynamic Behavior of a Bi-Cable Ropeway with Modal Bases
}

\author{
Gabriel Hurel, Jérôme Laborde, Louis Jezequel
}

\section{To cite this version:}

Gabriel Hurel, Jérôme Laborde, Louis Jezequel. Simulation of the Dynamic Behavior of a BiCable Ropeway with Modal Bases. Topics in Modal Analysis \& Testing, Volume 9, pp.43-54, 2019, 10.1007/978-3-319-74700-2_5. hal-02065316

\section{HAL Id: hal-02065316 https://hal.science/hal-02065316}

Submitted on 9 Nov 2020

HAL is a multi-disciplinary open access archive for the deposit and dissemination of scientific research documents, whether they are published or not. The documents may come from teaching and research institutions in France or abroad, or from public or private research centers.
L'archive ouverte pluridisciplinaire HAL, est destinée au dépôt et à la diffusion de documents scientifiques de niveau recherche, publiés ou non, émanant des établissements d'enseignement et de recherche français ou étrangers, des laboratoires publics ou privés. 


\title{
Simulation of the dynamic behavior of a bi-cable ropeway with modal bases
}

\author{
PhD Gabriel Hurel (Gabriel.Hurel@ec-lyon.fr), \\ Ing. Jerome Laborde and Pr. Louis Jézéquel \\ Ecole Centrale de Lyon, LTDS, \\ 36 avenue Guy de Collongue, 69134 Ecully, France
}

Published on July 5, 2018

doi:10.1007/978-3-319-74700-2_5

\begin{abstract}
This article presents the building of a dynamic model of a bi-cable ropeway. Thanks to the assumption of quasi-static advance of the vehicles, the calculation is performed step by step. At each step, a transient linearized dynamic solution is calculated around the quasi-static equilibrium using modal bases. The ropeway system is substructured considering the different elements: track rope, hauling rope and vehicles represented by pendulums. The results of time integration give the accelerations felt by the passengers according to the load case.
\end{abstract}

Keywords ropeway, dynamic, modal basis, substructuring, quasi-static

\section{Introduction}

\section{$1.1 \quad$ Problematic}

In order to improve mobility in cities, new types of public transport are investigated. In particular, ropeway is highlighted because it presents several benefits. First, the cost of building is relatively low compared to subway or tramway. It only needs to build stations and some pylons. The space used by the infrastructure on the floor is very low: unlike bus and tramway, the ropeway is not in the way of streets. Moreover, the speed of displacement is quite high and the throughput is similar to that of a tramway. Finally, this is one of the most secured type of transport. The rate of injuries and deaths per passengers is the lowest of all types of transportation. On the other side, ropeways have drawbacks. The regulation can be an obstacle to the proximity between the cabins and habitation buildings. In fact, the ropeway can create some discomfort for the neighborhood. The noise and the obstruction of the view can degrade the comfort of residents. It might also have some problems for the comfort of the passengers. The comfort criteria are thermic, acoustic and vibratory. Within the I2TC project, the objective of this article is to provide a tool able to solve dynamic and vibratory issues of an urban ropeway. In fact, the dynamic requirements for public transports are higher than touristic installations. For this, a dynamic modelling of a ropeway line is performed. Thus we aim to understand the dynamic behavior of the line and be able to predict the acceleration felt by passengers. The sensitive parameters can be found and an optimization can be performed. 


\subsection{The 3S cable-car}

One of the technologies considered for urban transportation using ropes is the 3S cable car. As its name indicates, the number of cables is three. Two of them are track ropes which sustain the vehicles. They are fixed and the cabins are rolling on them like a train on a railroad. The third cable is the hauling rope. Its role is to pull the vehicles along the line. This cable is moving at the same speed as the vehicles, since they are clamped on it. The track ropes are embedded in the first and the last station. Their tension is tuned few times in the year in order to compensate the thermal expansion. In service the tension is varying mainly due to the load of vehicles. The tension of the hauling rope is kept constant at the first station by a hydraulic cylinder. The tension at the last station is imposed by the engine to haul the vehicles. The vehicles are composed of three main parts: the cabin, where the passengers are, is hanged to a carriage by a hanger. The hanger is fixed to the carriage by a damped revolute joint. The carriage is fixed to the hauling rope by a clamp and it is rolling on both track ropes.

\section{$1.3 \quad$ Bibliography}

The theory of vibration of a cable was mainly developed by Irvine [1] in the seventies. He developed analytical models able to calculate the shapes and the frequencies of the first eigenmodes of a cable with a small sag. Sack [2], Simpson [3] and Triantafyllou [4] studied the vibrations of a cable in translation. Sergev and Iwan [5] were ones of the first to study a cable with masses. They represent a taut cable by a succession of segment. Rosenthal [6] used an iterative method to find the modes of a cable with sag and masses. Cheng and Perkins [7] calculated the eigenmodes when the position of the mass is varying from one end of the cable to the other. Al-Qassab [8] computed these eigenmodes with a Galerkin method. The first model of a ropeway is certainly that of Portier [9]. The vehicles are represented by pendulums and the cable by springs. The results are compared with experiments. Brownjohn [10] built a model with a small sag cable to modelize the ropeway of Singapore. Sofi [11] represented the cabins with damped oscillators.

\section{Model building}

\section{$2.1 \quad$ Presentation}

The proposed model shown in figure 1 corresponds to a span between two pylons. Since the model is planar, only the half of the system is considered: one track rope, a half of the hauling rope and half of the vehicles. Both pylons are assumed to be infinitely rigid. The cables are assumed to be elastic, with a mass. Because of low angles of bending, the bending rigidity is neglected.

Each vehicle is modelled by a pendulum as shown in figure 1. At the top, the carriage is represented by a point mass. It is in sliding contact with the track rope and clamped on the hauling rope. The hanger is attached to the carriage by a damped cylindrical joint. It has a rotational inertia and a longitudinal stiffness. Another point mass represents the cabin. In this model, dynamic effects that could occur between cables and the atmosphere are not considered.

\subsection{Assumptions}

Considering the studied system, two strong assumptions are made. First, the speed of the vehicles $C$ is low enough to consider the motion of the vehicles have no dynamic effect on the system. In other words, the cabins are slow enough to consider their advance as quasistatic. The system can be represented by a succession of static equilibriums. To validate this assumption, the results of a simple model of a mass moving on a taut cable is compared 


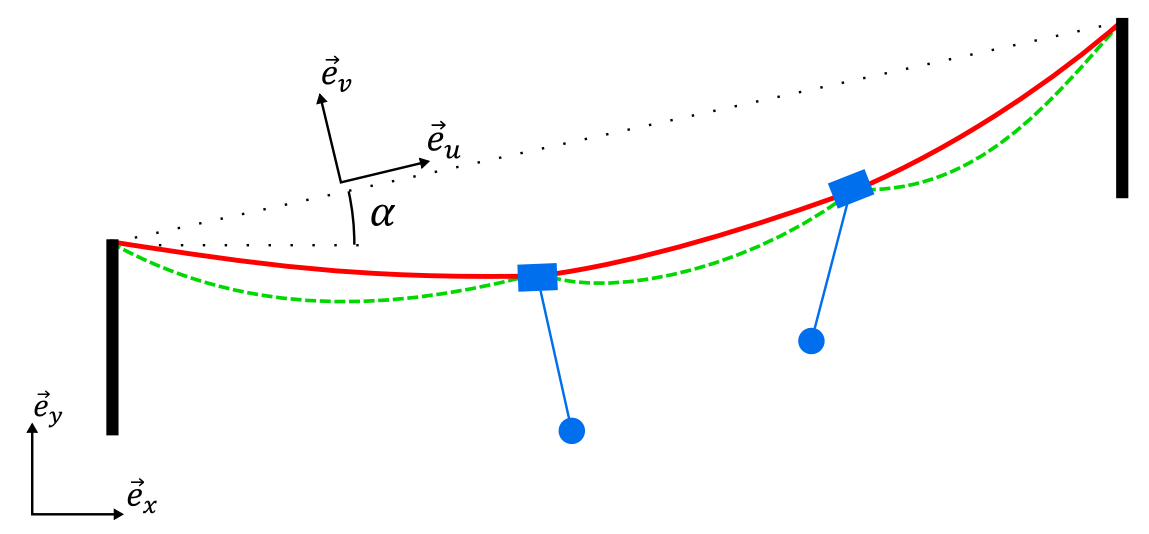

Figure 1: Model of the 3S cable car (red line: track rope, green line: hauling rope, circle: carriage, square: cabin, blue line: hanger)

with the quasi-static solution. Figure 2 shows the state of the cable when the mass is at the middle of the cable for three values of speed: a low speed similar to the speed of the cable car vehicles speed, a high speed similar to the speed of high speed trains and a transitional speed. The solid line represents the quasi-static solution. In every cases, the inertial forces tend to separate the cable from its static shape but in the high speed case these forces are higher so the error on the displacement cannot be neglected.

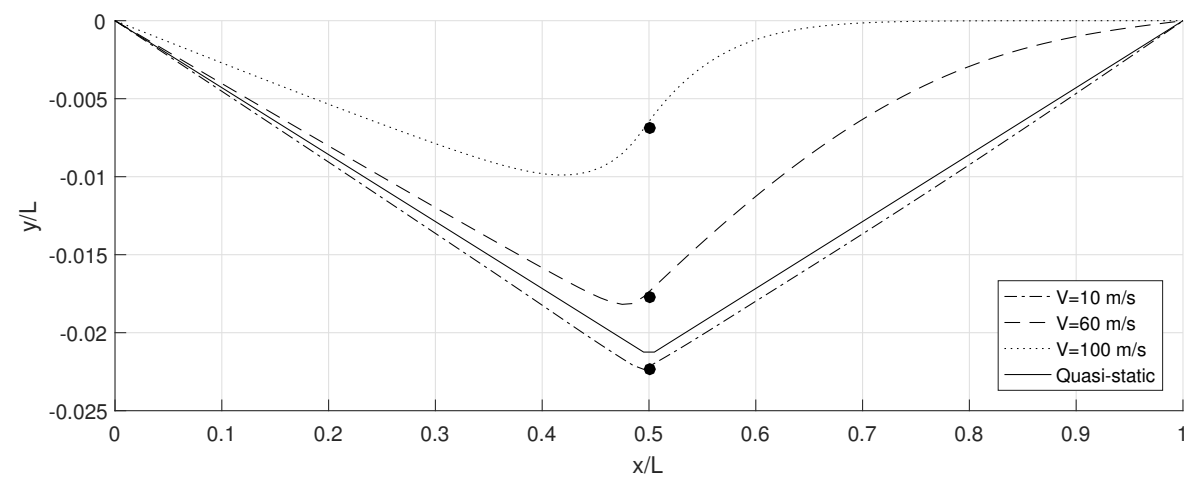

Figure 2: Comparison of the shape of the cable for three different speeds of the mass and the quasi static solution

At low speed $(10 \mathrm{~m} / \mathrm{s})$, figure 3 shows that the average error $E$ on the displacement is below $1 \%$.

$$
E=\frac{1}{T} \int_{0}^{T} \frac{1}{L} \int_{0}^{L}\left|\frac{u_{R}-u}{u_{R}}\right| \mathrm{d} x \mathrm{~d} t
$$

Where $T$ is the period required by the mass to travel on the cable, $L$ is the length of the cable, $u_{R}$ is the reference displacement of the cable and $u$ is the calculated displacement. A correction of the quasi static solution can be made by adding the inertial forces of the mass and the cable calculated with the succession of equilibrium states. The results with this correction shown in red line on figure 3 are better, in particular at speeds between 10 and $60 \mathrm{~m} \mathrm{~s}^{-1}$.

Around these static equilibriums, we assume the dynamic displacement is small enough to linearize the behavior equations. This important assumption allows us to project the problem on the eigenmodes of the structure. 


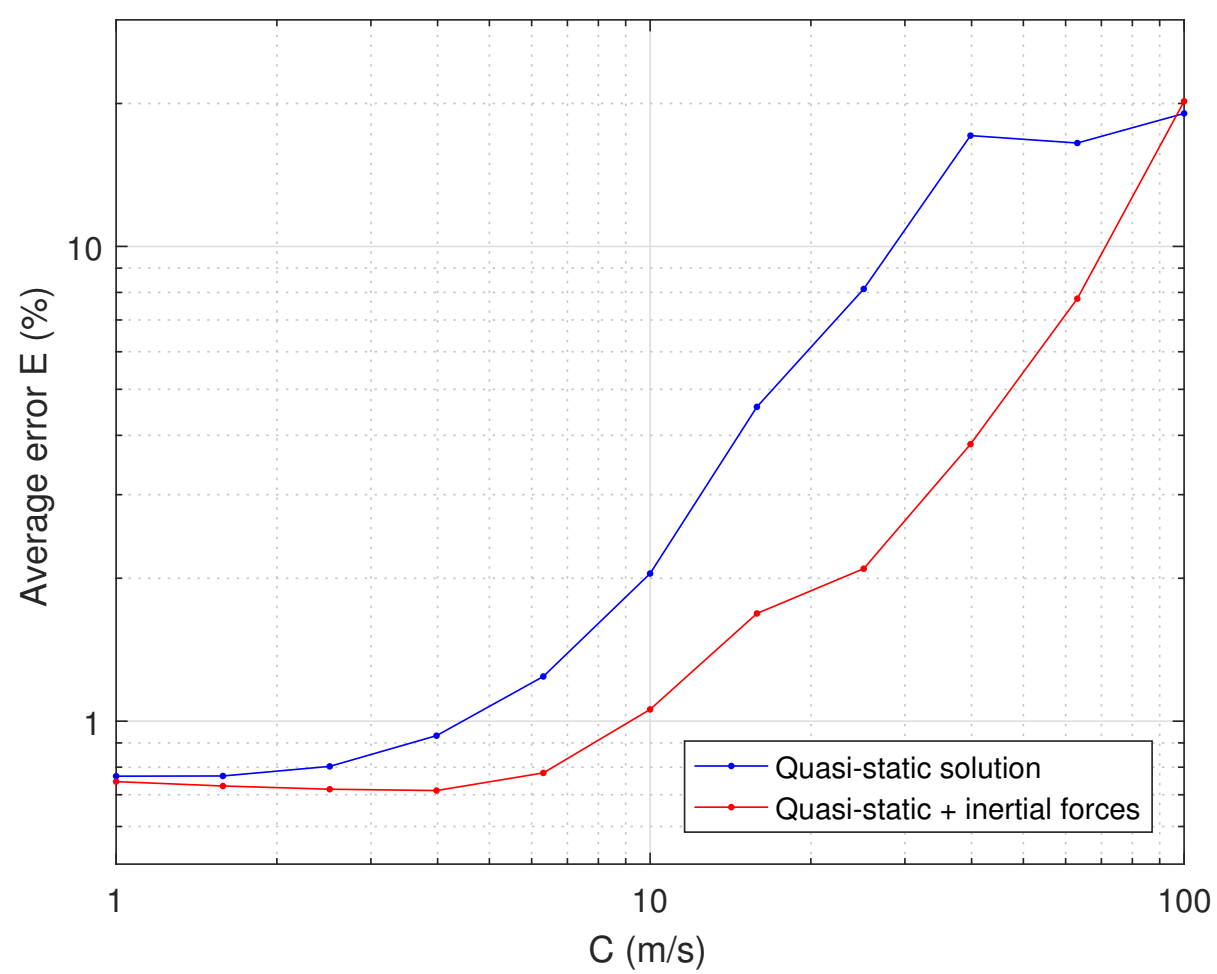

Figure 3: Average error with the quasi static assumption for different speeds of the mass

\subsection{Computation strategy}

Thanks to the first assumption, the computation is performed step by step. The advance of the vehicles is discretized. Each step corresponds to a position of the whole of vehicles along the cables. At each step a non-linear static equilibrium will be computed. Under the assumption of small dynamic displacement, linear eigenmodes of each cable part are calculated, taking into account the position of the vehicles and parameters of the static equilibrium: shape and tension of the cables. The eigenmodes of the entire track rope constitute a modal basis on which the behavior equations are projected. A part of the hauling rope is the portion between two vehicles or between a vehicle and a pylon. The extremity of each part of the hauling rope are not always stationary but may be linked to the vehicles. Static response modes have to be added to the natural modes. The behavior of the pendulums embodying the vehicles are considered as non-linear to keep a good coupling with cables. After assembling all parts: reduced track rope, reduced hauling rope parts and vehicles, a time integration is performed at each step. The initial conditions of a step are provided by the final state of the previous step. The global behavior of the system is given by the sum of static and dynamic results.

\section{Static equilibrium}

\subsection{Static equation of a cable}

The force on the elementary portion $\mathrm{d} s$ at the position $(x, y)$ are the weight and the tension on each side:

$$
\mu \mathrm{d} s \vec{g}+\vec{T}(x)+\vec{T}(x+\mathrm{d} x)=\overrightarrow{0}
$$


Where $\mu$ is the linear mass, $\vec{g}$ the gravitational acceleration and $T(x)$ the tension of the cable at the global coordinate $x$. The vector equation 2 is projected on $x$ and $y$ axes:

$$
\begin{aligned}
& \frac{\mathrm{d} T_{x}(x)}{\mathrm{d} x}=0 \\
& \frac{\mathrm{d} T_{y}(x)}{\mathrm{d} x}=\mu g \frac{\mathrm{d} s}{\mathrm{~d} x}
\end{aligned}
$$

The linear mass $\mu$ is not constant because of the elastic strain:

$$
\mu=\frac{\mu_{0}}{1+\frac{T}{E S}}
$$

Where $E$ and $S$ are the Young modulus and the area of the section of the cable respectively. In the ropeways case, the tension $T$ is negligible compared to the product $E S$. The order of magnitude of the ratio is about $5 \times 10^{-3}$. So we consider $\mu$ as a constant. The equations 3 and 4 give us the differential equation:

$$
\frac{\mathrm{d}^{2} y(x)}{\mathrm{d} x^{2}}=\frac{1}{\tau} \sqrt{1+\left(\frac{\mathrm{d} y(x)}{\mathrm{d} x}\right)^{2}}
$$

The constant $\tau$ is homogeneous to a length and equal to $\frac{T_{x}}{\mu g}$. The solution of the equation 6 corresponds to a catenary curve:

$$
y(x)=\tau \cosh \left(\frac{x}{\tau}+K_{1}\right)+K_{2}
$$

The constants $K_{1}$ and $K_{2}$ are calculated with the boundary conditions for each part of the cable. The expression of the tension of the cable is given by:

$$
T(x)=T_{x} \cosh \left(\frac{x}{\tau}+K_{1}\right)
$$

\subsection{Equilibrium of a vehicle}

The forces exerted on a vehicle with a mass $M$ at the abscissa $x_{M}$ are the gravitation, the tension of the hauling rope $T_{H}$ on the left side and on the right side and the reaction of the track rope $N$ :

$$
M \vec{g}+\vec{T}_{H}^{L}(x)+\vec{T}_{H}^{R}(x)+\vec{N}=\overrightarrow{0}
$$

The contact between the vehicle and the track rope is frictionless, hence the force $\vec{N}$ is orthogonal to the track rope. However the angle of the cable on the $\varphi_{L}$ is not the same as the angle on the right side $\varphi_{R}$. The angle $\varphi_{M}$ of the force $\vec{N}$ is chosen as the average of the angles of the track rope:

$$
\varphi_{M}=\frac{\varphi_{L}+\varphi_{R}}{2}
$$

The equilibrium of the point of the track rope in contact with the vehicle is given by:

$$
\vec{T}_{L}(x)+\vec{T}_{T}(x)=\vec{N}
$$

The projection on the direction orthogonal to $\vec{N}$ shows that the tension of the track rope $T_{T}$ is equal on the left and on the right of the vehicle. 


\subsection{Equilibrium of the span}

In the same way as the linear mass, we consider the length of the track rope $L_{c}$ is not varying because of its elasticity, hence it is constant:

$$
\begin{aligned}
L_{c} & =\int_{A}^{B} \mathrm{~d} s=\int_{x_{A}}^{X_{B}} \sqrt{1+\left(\frac{\mathrm{d} y_{c}(x)}{\mathrm{d} x}\right)^{2}} \mathrm{~d} x=\sum_{i=1}^{n+1} \int_{x_{i-1}}^{X_{i}} \tau_{c i} \frac{\mathrm{d}^{2} y_{c}(x)}{\mathrm{d} x^{2}} \mathrm{~d} x \\
& =\sum_{i=1}^{n+1} \int_{x_{i-1}}^{X_{i}} \cosh \left(\frac{x}{\tau_{c i}}+K_{1 c i}\right)
\end{aligned}
$$

The number $n$ is the number of vehicles on the span. Consequently there are $n+1$ parts on each cables in the span. The equations of the equilibrium of the vehicles 9 and 11 and the equation 12 are implemented in a nonlinear solver to determine the constants $K_{1 i}$ and $K_{2 i}$ of each cable part.

\subsection{Results}

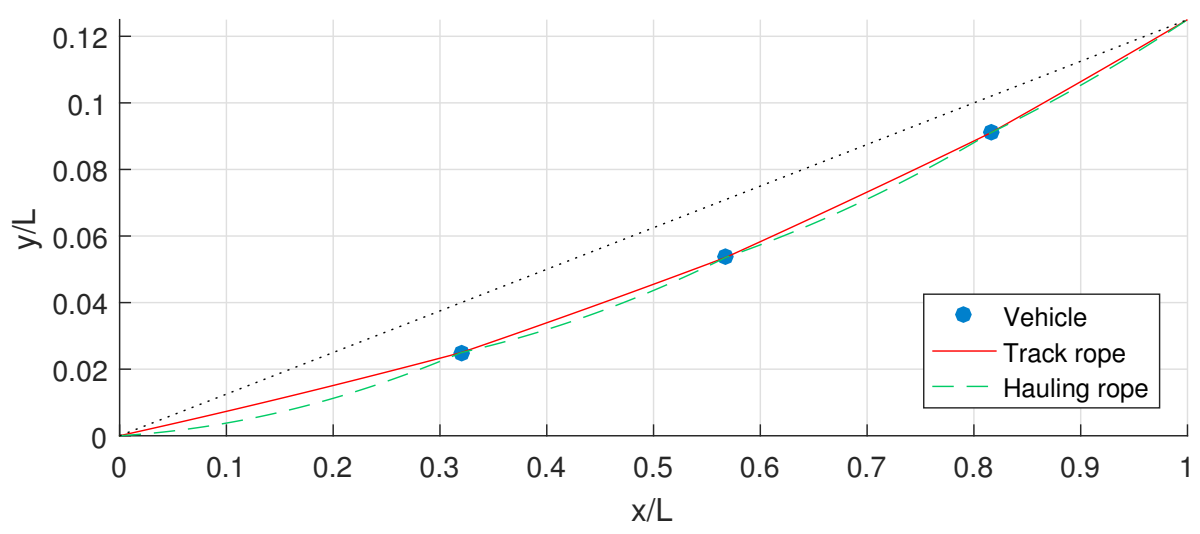

Figure 4: Shape of a span with three vehicles.

Figure 4 shows the shape of the cables of a span with three vehicles in a certain position. Because $\tau_{c i}>\tau_{h i}$, the sag of the hauling rope is more important than the sag of the track rope. The tension of both cables are shown in figure 5 for the same position of the vehicles. The tension of the track rope is continuous whereas the tension of the hauling rope has discontinuities at each vehicle.

\section{Dynamic response}

The objective is to predict the dynamic response of the system while the vehicles are moving forward. Hence, we need to perform transient simulations. First, this kind of simulation is computationally expensive. The less number of degrees of freedom there is, the faster the computations are. Secondly we are interested by the low frequency dynamic phenomena of the system. Hence we choose to make a modal reduction of the cables. This method allows to take a small amount of generalized degrees of freedom while the low frequency phenomena are well described. However, at each step, the system is changing. The modal bases have to be recalculated. The computation of modes has to be fast. The structure of a cable car is characterized by elongated parts with very small interfaces represented by points in the model. The substructuring method appears as a good solution to our problem. The 

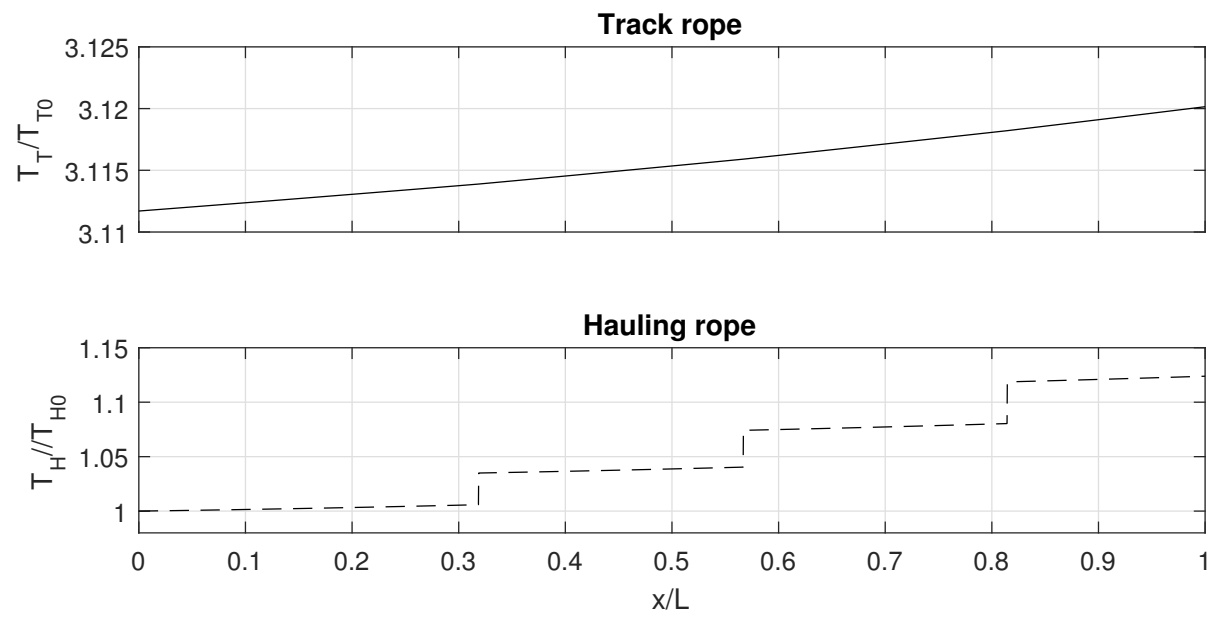

Figure 5: Tension of the ropes

computation of the eigenmodes of each part of the cable separately is not time-expensive and the representation is relatively good.

\subsection{Track rope}

The dynamic equations of an inclined cable with a mass density are given by Irvine [1]:

$$
\begin{aligned}
& \mu\left(\frac{\partial^{2} u(t, s)}{\partial t^{2}}-g \sin \alpha\right)=\frac{\partial}{\partial s}\left[T(s, t)\left(\frac{\partial u(t, s)}{\partial s}+\frac{\mathrm{d} U(s)}{\mathrm{d} s}\right)\right] \\
& \mu\left(\frac{\partial^{2} v(t, s)}{\partial t^{2}}+g \cos \alpha\right)=\frac{\partial}{\partial s}\left[T(s, t)\left(\frac{\partial v(t, s)}{\partial s}+\frac{\mathrm{d} V(s)}{\mathrm{d} s}\right)\right]
\end{aligned}
$$

At the curvilinear abscissa $s$, the static position of the point of the cable is described by the coordinates $U(s)$ and $V(s)$ in the frame of reference $\left(\vec{e}_{u}, \vec{e}_{v}\right)$. The coordinates $u(s, t)$ and $v(s, t)$ are the dynamic displacements of the point at the curvilinear abscissa $s$ at the time $t$. The tension $T$ can be split up in a static and a dynamic contribution:

$$
T(s, t)=T_{S}(s)+T_{D}(s, t)
$$

The equations 13 and 14 describe the dynamic and static behavior. The static terms can be subtracted:

$$
\begin{aligned}
& \mu \frac{\partial^{2} u}{\partial t^{2}}=\frac{\partial}{\partial s}\left[T_{S}(s) \frac{\partial u(t, s)}{\partial s}+T_{D}(s, t)\left(\frac{\partial u(t, s)}{\partial s}+\frac{\mathrm{d} U(s)}{\mathrm{d} s}\right)\right] \\
& \mu \frac{\partial^{2} v}{\partial t^{2}}=\frac{\partial}{\partial s}\left[T_{S}(s) \frac{\partial u(t, s)}{\partial s}+T_{D}(s, t)\left(\frac{\partial v(t, s)}{\partial s}+\frac{\mathrm{d} V(s)}{\mathrm{d} s}\right)\right]
\end{aligned}
$$

Previously, we assumed the global length of the cable is constant because the tension was neglected compared to the stiffness. However, in order to express the dynamic tension, we consider the local strain of the cable $\varepsilon(s, t)$ with the Green-Lagrange deformation:

$$
\begin{aligned}
T_{D}(s, t) & =E S \varepsilon(s, t) \\
& =E S\left[\frac{\partial u(s, t)}{\partial s} \frac{\mathrm{d} U(s)}{\mathrm{d} s}+\frac{\partial v(s, t)}{\partial s} \frac{\mathrm{d} V(s)}{\mathrm{d} s}+\frac{1}{2}\left(\left(\frac{\partial u(s, t)}{\partial s}\right)^{2}+\left(\frac{\partial v(s, t)}{\partial s}\right)^{2}\right)\right]
\end{aligned}
$$


The expression of dynamic tension 15 is replaced in the equations 13 and 14 . The equations are linearized neglecting the terms in $u$ and $v$ with an order greater or equal to two:

$$
\begin{aligned}
\mu \frac{\partial^{2} u}{\partial t^{2}}= & \left(\frac{\mathrm{d} T_{S}}{\mathrm{~d} s}+2 E S \frac{\mathrm{d} U}{\mathrm{~d} s} \frac{\mathrm{d}^{2} U}{\mathrm{~d} s^{2}}\right) \frac{\partial u}{\partial s}+\left(T_{S}+E S\left(\frac{\mathrm{d} U}{\mathrm{~d} s}\right)^{2}\right) \frac{\partial^{2} u}{\partial s^{2}} \\
& +E S\left(\frac{\mathrm{d} U}{\mathrm{~d} s} \frac{\mathrm{d}^{2} V}{\mathrm{~d} s^{2}}+\frac{\mathrm{d} V}{\mathrm{~d} s} \frac{\mathrm{d}^{2} U}{\mathrm{~d} s^{2}}\right) \frac{\partial v}{\partial s}+E S \frac{\mathrm{d} U}{\mathrm{~d} s} \frac{\mathrm{d} V}{\mathrm{~d} s} \frac{\partial^{2} v}{\partial s^{2}} \\
\mu \frac{\partial^{2} v}{\partial t^{2}}= & \left(\frac{\mathrm{d} T_{S}}{\mathrm{~d} s}+2 E S \frac{\mathrm{d} V}{\mathrm{~d} s} \frac{\mathrm{d}^{2} V}{\mathrm{~d} s^{2}}\right) \frac{\partial v}{\partial s}+\left(T_{S}+E S\left(\frac{\mathrm{d} V}{\mathrm{~d} s}\right)^{2}\right) \frac{\partial^{2} v}{\partial s^{2}} \\
& +E S\left(\frac{\mathrm{d} U}{\mathrm{~d} s} \frac{\mathrm{d}^{2} V}{\mathrm{~d} s^{2}}+\frac{\mathrm{d} V}{\mathrm{~d} s} \frac{\mathrm{d}^{2} U}{\mathrm{~d} s^{2}}\right) \frac{\partial u}{\partial s}+E S \frac{\mathrm{d} U}{\mathrm{~d} s} \frac{\mathrm{d} V}{\mathrm{~d} s} \frac{\partial^{2} u}{\partial s^{2}}
\end{aligned}
$$

Because the track rope is only in frictionless contact with the vehicles, the modes of the whole span of the cable are calculated. However, in order to better represent its behavior, masses corresponding to the vehicles are added for the computation of eigenmodes. These masses are removed before the reduction and replaced by pendulums for the assembly step. The modes are computed with a finite difference algorithm. Figure 6 shows the variations of the frequencies of the first eigenmodes with respect to the positions of the vehicles. The frequency of the first mode of the cable without any vehicle is $f_{0}$.

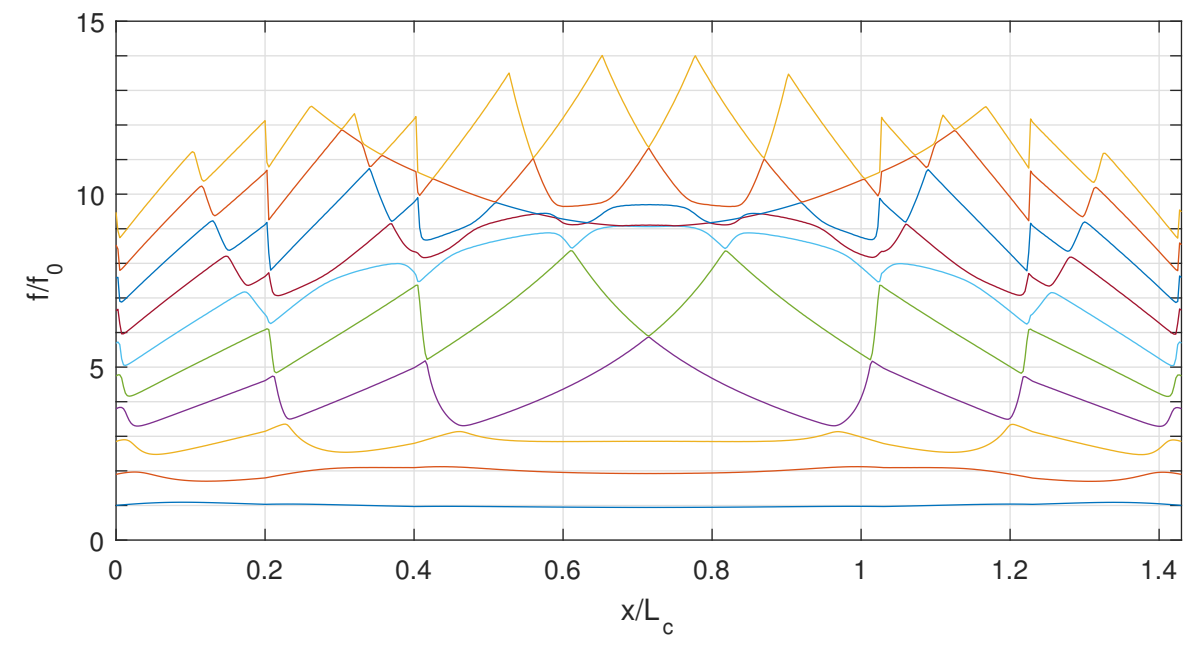

Figure 6: Frequencies of the first ten modes of the track rope with the vehicles as punctual masses with respect to the progression of the track rope

\subsection{Hauling rope}

The hauling rope is moving with the vehicle with a velocity $C$. Convection terms appear in its dynamic equations $[2,3]$ :

$$
\begin{array}{r}
\mu\left(\frac{\partial^{2} u}{\partial t^{2}}+2 C \frac{\partial^{2} u}{\partial t \partial s}+C^{2} \frac{\partial^{2} u}{\partial s^{2}}+\frac{\mathrm{d} C}{\mathrm{~d} t} \frac{\partial u}{\partial s}\right)=\mu g \sin \alpha+\frac{\partial}{\partial s}\left[T\left(\frac{\partial u}{\partial s}+\frac{\mathrm{d} U}{\mathrm{~d} s}\right)\right] \\
\mu\left(\frac{\partial^{2} v}{\partial t^{2}}+2 C \frac{\partial^{2} v}{\partial t \partial s}+C^{2} \frac{\partial^{2} v}{\partial s^{2}}+\frac{\mathrm{d} C}{\mathrm{~d} t} \frac{\partial v}{\partial s}\right)=-\mu g \cos \alpha+\frac{\partial}{\partial s}\left[T\left(\frac{\partial v}{\partial s}+\frac{\mathrm{d} V}{\mathrm{~d} s}\right)\right]
\end{array}
$$


By considering a constant speed of the cable, subtracting the static terms and replacing the dynamic tension with the Green-Lagrange strain, we obtain:

$$
\begin{aligned}
\mu \frac{\partial^{2} u}{\partial t^{2}}+2 \mu C \frac{\partial^{2} u}{\partial t \partial s}= & \left(\frac{\mathrm{d} T_{S}}{\mathrm{~d} s}+2 E S \frac{\mathrm{d} V}{\mathrm{~d} s} \frac{\mathrm{d}^{2} V}{\mathrm{~d} s^{2}}-\mu \frac{\mathrm{d} C}{\mathrm{~d} t}\right) \frac{\partial u}{\partial s}+\left(T_{S}+E S\left(\frac{\mathrm{d} V}{\mathrm{~d} s}\right)^{2}-\mu C^{2}\right) \frac{\partial^{2} u}{\partial s^{2}} \\
& +E S\left(\frac{\mathrm{d} U}{\mathrm{~d} s} \frac{\mathrm{d}^{2} V}{\mathrm{~d} s^{2}}+\frac{\mathrm{d} V}{\mathrm{~d} s} \frac{\mathrm{d}^{2} U}{\mathrm{~d} s^{2}}\right) \frac{\partial v}{\partial s}+E S \frac{\mathrm{d} U}{\mathrm{~d} s} \frac{\mathrm{d} V}{\mathrm{~d} s} \frac{\partial^{2} v}{\partial s^{2}} \\
\mu \frac{\partial^{2} v}{\partial t^{2}}+2 \mu C \frac{\partial^{2} v}{\partial t \partial s}= & \left(\frac{\mathrm{d} T_{S}}{\mathrm{~d} s}+2 E S \frac{\mathrm{d} V}{\mathrm{~d} s} \frac{\mathrm{d}^{2} V}{\mathrm{~d} s^{2}}-\mu \frac{\mathrm{d} C}{\mathrm{~d} t}\right) \frac{\partial v}{\partial s}+\left(T_{S}+E S\left(\frac{\mathrm{d} V}{\mathrm{~d} s}\right)^{2}-\mu C^{2}\right) \frac{\partial^{2} v}{\partial s^{2}} \\
& +E S\left(\frac{\mathrm{d} U}{\mathrm{~d} s} \frac{\mathrm{d}^{2} V}{\mathrm{~d} s^{2}}+\frac{\mathrm{d} V}{\mathrm{~d} s} \frac{\mathrm{d}^{2} U}{\mathrm{~d} s^{2}}\right) \frac{\partial u}{\partial s}+E S \frac{\mathrm{d} U}{\mathrm{~d} s} \frac{\mathrm{d} V}{\mathrm{~d} s} \frac{\partial^{2} u}{\partial s^{2}}
\end{aligned}
$$

The mixed partial term creates an antisymmetric damping, resulting in complex modes. The shape of the first mode of a portion of a hauling rope of $100 \mathrm{~m}$ is represented on figure 7 for two values of $C$.

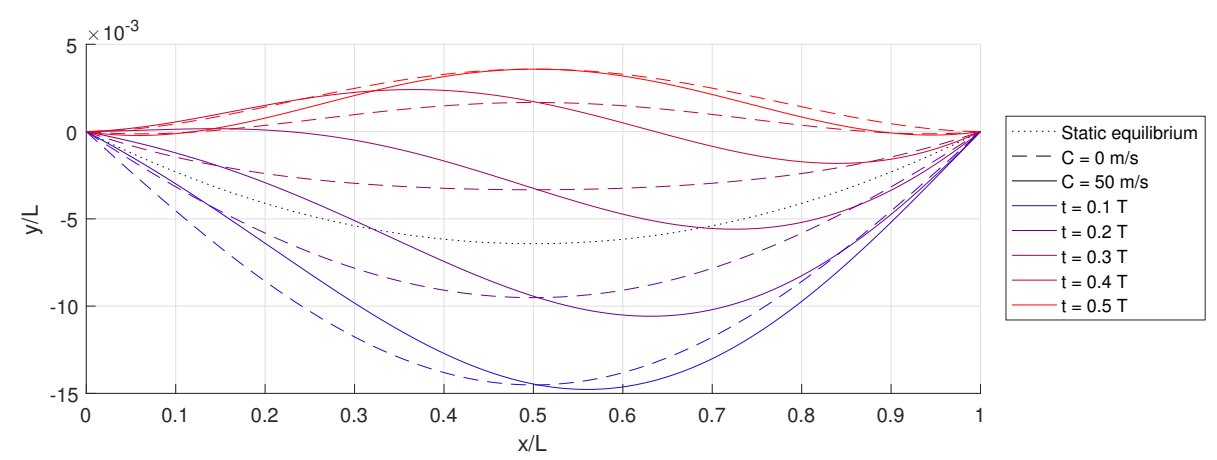

Figure 7: Shapes of the first mode of a portion of a hauling rope for a speed of 0 and $50 \mathrm{~m} \mathrm{~s}^{-1}$ at different instants corresponding to factions of the period $T$.

\subsection{Vehicle}
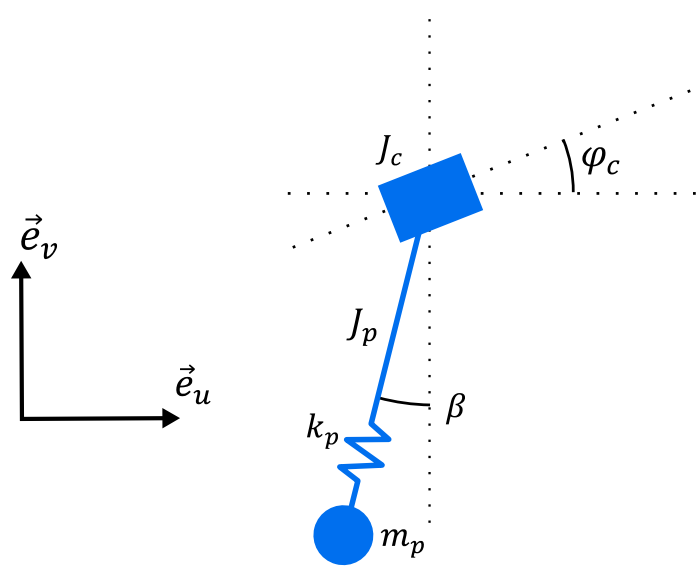

Figure 8: Model of a vehicle

Each vehicle is modelled by a pendulum consisting of a carriage a hanger and a point 
mass. The kinetic and potential energies $K$ and $U$ are expressed by:

$$
\begin{aligned}
K & =\frac{1}{2} m_{c}\left(\dot{u}_{c}^{2}+\dot{v}_{c}^{2}\right)+\frac{1}{2} J_{c} \dot{\varphi}_{c}^{2}+\frac{1}{2} J_{p} \dot{\beta}^{2} \\
U & =\frac{1}{2} k_{p} \delta^{2}+g\left[m_{c}\left(u_{c} \sin \alpha+v_{c} \cos \alpha\right)+m_{p}\left(u_{c} \sin \alpha+v_{c} \cos \alpha-\left(l_{p}+\delta \cos (\beta+\alpha)\right)\right]\right.
\end{aligned}
$$

Where $m_{c}, u_{c}, v_{c}, J_{c}$ and $\varphi_{c}$ are the masses, the displacement in both directions, the moment of inertia and the rotation angle of the carriage respectively. The variables $\delta, l_{p}, J_{p}, \beta_{p}$ are the elongation, the length, the moment of inertia and the angle of the hanger respectively and $m_{p}$ is the mass of the cabin. By applying the Lagrange equations, the dynamic matrix equation is obtained:

$$
\mathbf{M}_{v} \ddot{X}+\mathbf{C}_{v} \dot{X}+\mathbf{K}_{v} V=F_{g}+F_{\mathrm{NL}}(\beta, \dot{\beta}, \dot{\delta})
$$

The vector $X$ is the vector of the five degrees of freedom of the pendulum:

$$
X=\left[\begin{array}{lllll}
u_{c} & v_{c} & \varphi_{c} & \beta & \delta
\end{array}\right]^{T}
$$

The matrix of mass $\mathbf{M}_{v}$ and the vector of gravitation force $F_{g}$ are not constant but depend on the $\beta$ angle. The non-linear terms are gathered in the nonlinear force vector:

$$
F_{\mathrm{NL}}=\left[\begin{array}{c}
-m_{p}\left(l_{p} \dot{\beta}^{2} \sin \beta-2 \dot{\beta} \dot{\delta} \cos \beta\right) \\
-m_{p}\left(l_{p} \dot{\beta}^{2} \cos \beta+2 \dot{\beta} \dot{\delta} \sin \beta\right) \\
0 \\
-2 m_{p} l_{p} \dot{\beta} \dot{\delta} \\
m_{p} l_{p} \dot{\beta}^{2}
\end{array}\right]
$$

\subsection{Time integration}

At each step $p$, the track rope, the portions of the hauling rope and the pendulums are assembled to make the matrices of mass $\mathbf{M}_{p}$, damping $\mathbf{C}_{p}$ and stiffness $\mathbf{K}_{p}$. The generalized degrees of freedom $Q_{p}$ associated are a combination of the modes of the carrying and hauling ropes and the physical degrees of freedom of each pendulum $X$. Then, a transient calculation is performed on a duration of the step $\Delta t$ :

$$
\mathbf{M}_{p} \ddot{Q}_{p}+\mathbf{C}_{p} \dot{Q}+\mathbf{K}_{p} Q_{p}=F_{Q g}+F_{Q \mathrm{NL}}(Q)+F_{I}
$$

The external forces $F_{Q g}, F_{Q N \mathrm{~L}}(Q)$ and $F_{I}$ are respectively the gravitational forces, the nonlinear terms of the pendulums and the inertial forces due to the quasi-static assumption calculated with the succession of static equilibriums. The initial conditions of the transient calculation at the step $p$ are given by the previous step projected from the previous modal basis $\Phi_{p-1}$ to the current basis $\Phi_{p}$ :

$$
Q_{p}(0)=\Phi_{p}^{-1} \Phi_{p-1} Q_{p-1}(\Delta t)
$$

\section{Results}

\subsection{Start with an angle}

In this case, a train of three vehicles cross the span from left to right. Each vehicle arrives on the track rope with different angles: $10^{\circ}, 20^{\circ}$ and $30^{\circ}$. Figure 9.a shows the variations of the angles of the cabins. The acceleration felt by the passengers increases with the growth of the start angle. 


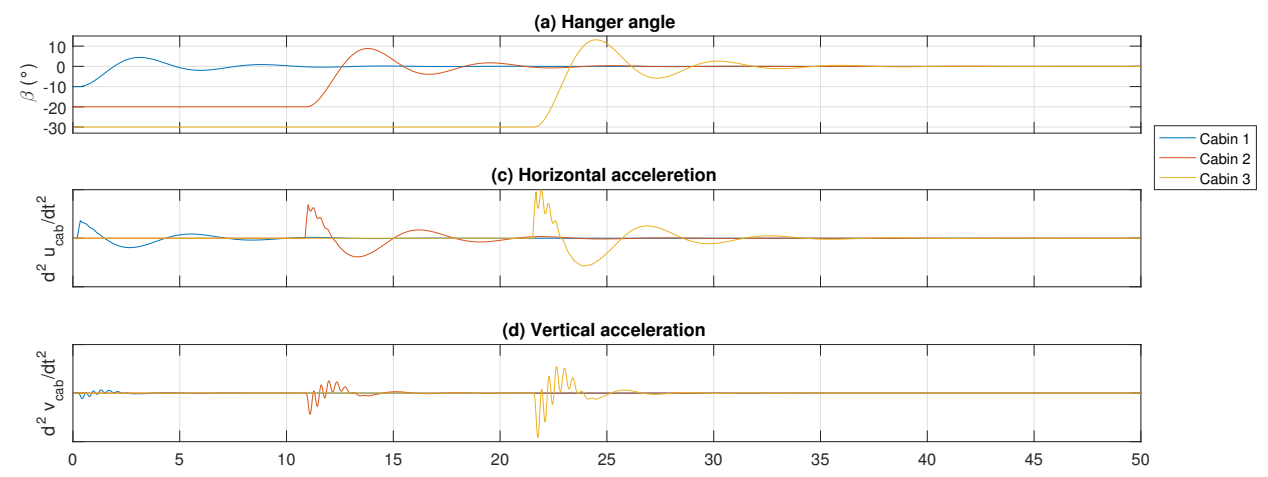

Figure 9: (a) Angles of the hangers of the vehicles (b) Longitudinal acceleration of the cabins (c) Vertical acceleration of the cabins

\subsection{Emergency braking}

The objective now is to simulate an emergency braking of the ropeway. With the quasi-static assumption, it is not possible to make a deceleration on the hauling rope because at each step, the vehicles are not moving in the model. To represent the deceleration, a braking force is applied on both extremity of the hauling rope. Figure 10 shows the accelerations felt by the passengers after the emergency braking.
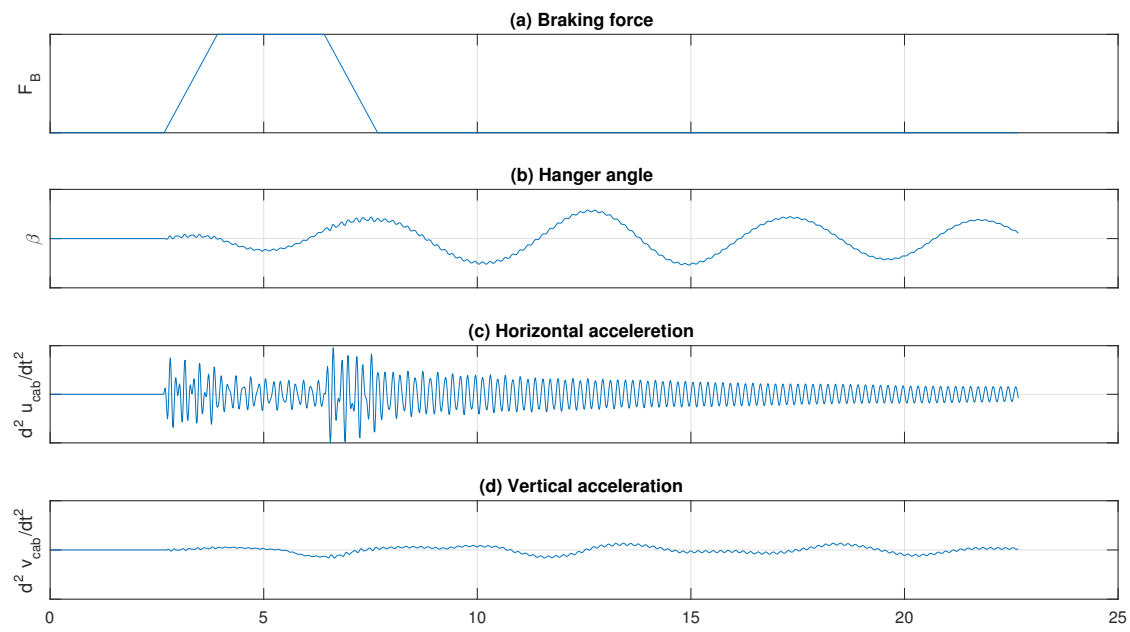

Figure 10: (a) Braking force (b) Angles of the hangers of the vehicle (c) Longitudinal acceleration of the cabin $(d)$ Vertical acceleration of the cabin

\section{Conclusion and perspectives}

In this article, a dynamic model of a ropeway is made. The assumptions of quasi-static advance of the cabins and small displacement allow to use a strategy of step by step computation. At each step, the quasi-static equilibrium is determined with a non-linear calculation. Then a modal basis of the track rope with masses is computed. The eigenmodes of each part of the hauling rope are calculated in considering its velocity. The vehicles are represented by 
non-linear pendulums. After assembling all the parts, a transient calculation is performed to know the dynamic behavior of the ropeway according to the load case. Two examples of load cases are given: a vehicle with a start angle and an emergency braking. The model could be improved by considering several spans, pylons as flexible structures and introducing the third dimension.

The I2TC project has been funded by the FUI I2TC, the Rhone-Alpes Region, the Ilede-France Region and the Paris Department in collaboration with the Ecole Centrale de Lyon, EIFFAGE, POMA, RATP, CD VIA, The Vibrant Project, the University of Paris 1 Pantheon-Sorbonne and the ENSTA.

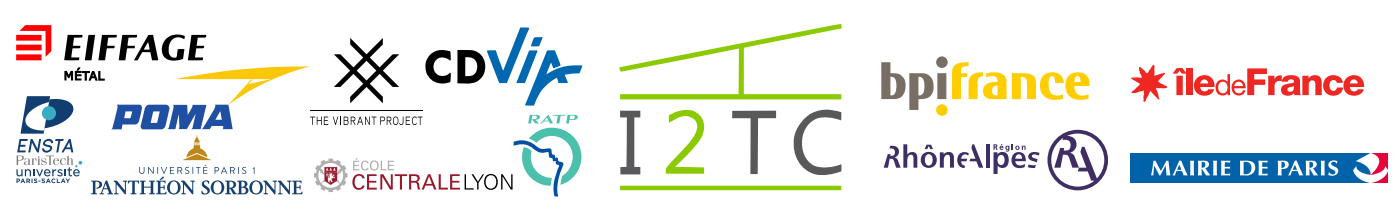

Figure 11: Contributors and funders

\section{References}

[1] H. M. Irvine, T. K. Caughey, and Gerald Beresford Whitham. "The linear theory of free vibrations of a suspended cable". Proceedings of the Royal Society of London. A. Mathematical and Physical Sciences 341.1626 (1974), pp. 299-315. DOI: 10.1098/rspa. 1974.0189 (cit. on pp. 2, 7).

[2] R A Sack. "Transverse oscillations in travelling strings". British Journal of Applied Physics 5.6 (1954), pp. 224-226. DOI: 10.1088/0508-3443/5/6/307 (cit. on pp. 2, 8).

[3] A. Simpson. "On the oscillatory motions of translating elastic cables". Journal of Sound and Vibration 20.2 (1972), pp. 177-189. DOI: 10.1016/0022-460X(72)90420-8 (cit. on pp. 2, 8).

[4] M.S. Triantafyllou. "The dynamics of translating cables". Journal of Sound and Vibration 103.2 (1985), pp. 171-182. DOI: 10.1016/0022-460X(85)90231-7 (cit. on p. 2).

[5] S. S. Sergev and W. D. Iwan. "The Natural Frequencies and Mode Shapes of Cables With Attached Masses". Journal of Energy Resources Technology 103.3 (1981), pp. 237-242. DOI: 10.1115/1.3230844 (cit. on p. 2).

[6] F. Rosenthal. "Vibrations of slack cables with discrete masses". Journal of Sound and Vibration 78.4 (1981), pp. 573-583. DOI: 10.1016/S0022-460X(81)80126-5 (cit. on p. 2).

[7] S.-P. Cheng and N. C. Perkins. "Free vibration of a sagged cable supporting a discrete mass". The Journal of the Acoustical Society of America 91.5 (1992), pp. 2654-2662. DOI: 10.1121/1.402973 (cit. on p. 2).

[8] M. Al-Qassab and S. Nair. "Wavelet-Galerkin method for the free vibrations of an elastic cable carrying an attached mass". Journal of Sound and Vibration 270.1-2 (2004), pp. 191-206. DOI: 10.1016/S0022-460X(03)00490-5 (cit. on p. 2).

[9] B. Portier. "Dynamic phenomena in ropeways after a haul rope rupture". Earthquake Engineering \&S Structural Dynamics 12.4 (1984), pp. 433-449. DOI: 10.1002 / eqe. 4290120402 (cit. on p. 2).

[10] James M.W. Brownjohn. "Dynamics of an aerial cableway system". Engineering Structures 20.9 (1998), pp. 826-836. DOI: 10.1016/S0141-0296(97)00113-2 (cit. on p. 2).

[11] Alba Sofi and Giuseppe Muscolino. "Dynamic analysis of suspended cables carrying moving oscillators". International Journal of Solids and Structures 44.21 (2007), pp. 6725-6743. DOI: 10.1016/j.ijsolstr.2007.03.004 (cit. on p. 2). 